\title{
Formes de présence et fonctions politiques des représentants en mission dans un microcosme urbain, l'exemple d'Amboise (Indre-et-Loire)
}

Anne Jollet

\section{(2) OpenEdition Journals}

Édition électronique

URL : https://journals.openedition.org/ahrf/763

DOI : 10.4000/ahrf.763

ISSN : 1952-403X

Éditeur :

Armand Colin, Société des études robespierristes

Édition imprimée

Date de publication : 1 décembre 2002

Pagination : 87-100

ISSN : 0003-4436

Référence électronique

Anne Jollet, « Formes de présence et fonctions politiques des représentants en mission dans un microcosme urbain, l'exemple d'Amboise (Indre-et-Loire) », Annales historiques de la Révolution française [En ligne], 330 | octobre-décembre 2002, mis en ligne le 17 avril 2008, consulté le 23 avril 2022. URL : http://journals.openedition.org/ahrf/763 ; DOI : https://doi.org/10.4000/ahrf.763 


\title{
FORMES DE PRÉSENCE ET FONCTIONS POLITIQUES DES REPRÉSENTANTS EN MISSION DANS UN MICROCOSME URBAIN, L'EXEMPLE D'AMBOISE (INDRE-ET-LOIRE)
}

\begin{abstract}
ANNE JOLLET
L'article cherche à montrer à travers l'étude de la vie politique d'une petite ville de Touraine combien les rapports de force politiques déterminant les prises de décision politique ont pu être complexes et mouvants, y compris au cceur de la période de la Terreur. Il observe notamment le rôle des représentants en mission dans le jeu de ces rapports de force locaux, représentants qui restent des individus de passage, en partie seulement au fait des enjeux des conflits locaux, représentants dont les pouvoirs sont mis en concurrence, entre ceux de divers représentants, mais aussi avec les pouvoirs d'autres institutions telles que l'Assemblée nationale ou le Comité de salut public. Cette approche permet de relire les rapports du local et du national, de la province et du pouvoir parisien dans le sens de l'existence d'une réelle marge d'autonomie du moins dans certains contextes - des forces locales, elles-mêmes diverses, qui savent aussi utiliser la diversité réelle des pouvoirs nationaux pour les amener à coups de courriers, de publications, de déplacements, à décider dans leur sens.
\end{abstract}

Mots clés : pouvoir local ; information; circulation; représentants en mission ; municipalité ; district ; Comité de surveillance ; Amboise ; Touraine ; Gerboin.

L'échelle de la petite ville apparaît bien adaptée à l'étude des interactions entre les multiples pouvoirs que la Révolution a mis en place. La multiplicité des pouvoirs n'est pas une chose nouvelle. Mais ce qui est plus caractéristique de la période révolutionnaire est la variation rapide de l'importance relative des différents pouvoirs, l'instabilité des rapports de force entre ces pouvoirs. Ces variations dans l'agencement des pouvoirs au niveau des diverses unités géographiques où ils s'exercent doivent à la fois au cadre réglementaire, aux inflexions qui sont données par le pouvoir central, et aux équilibres politiques locaux.

Annales historiques de la Révolution française - $2002-N^{\circ} 4$ [87 à 100] 
Dans la diversité de ces rapports de pouvoirs jouent, en particulier, les relations des pouvoirs locaux (municipalité, district, société populaire, comité de surveillance) avec les pouvoirs exogènes, pouvoirs départementaux, pouvoirs nationaux, Assemblée nationale, Comité de salut public, comités divers, Club des jacobins et les fameux représentants en mission.

Ce sont certaines des formes prises par ces interactions entre pouvoirs dans la ville et pouvoirs nationaux, expression concrète de ce que nous appelons de façon rapide les relations entre les provinces et Paris, que j'étudierai ici, en focalisant mon attention sur quelques épisodes entre l'automne 1793 et l'hiver 1794, moment de tension particulièrement aiguë liée à la guerre, moment de luttes exacerbées quant à la forme à donner au gouvernement révolutionnaire (1).

L'observatoire provincial est la petite ville d'Amboise, une ville d'environ 5000 habitants, chef-lieu de district du département d'Indre-etLoire, un morceau de province relativement peu éloigné de la capitale - à peine deux centaines de kilomètres (2). De par sa situation en bord de Loire, à une vingtaine de kilomètres par de bonnes routes du chef-lieu du département, Tours, de par sa situation sur un des ponts franchissant la Loire, sur l'ancienne route royale menant de Paris en Espagne, Amboise est un lieu de passage particulièrement intense. La situation de la ville est caractérisée aussi de façon conjoncturelle, mais importante pour notre propos, à partir du printemps 1793, par la proximité du front intérieur, du front de l'Ouest. C'est à ce titre qu'Amboise, arrière du front, est notamment visitée par des représentants nombreux en route vers l'Ouest. Et les habitants de la ville savent que, même s'ils ne sont pas physiquement présents, des représentants sont non loin, sollicitant les autorités locales et fournissant des interlocuteurs toujours susceptibles d'intervenir dans les affrontements locaux.

\section{Sollicitation par les acteurs locaux des représentants}

La ville d'Amboise connaît à l'automne 1793 de vives tensions politiques qui opposent le conseil municipal et l'administration du district. Ces tensions ne sont pas nouvelles. Le différénd est notamment devenu

(1) Moment qu'en d'autres temps, un historien comme Colin Lucas a pu appeler celui « du système de gouvernement de la Terreur *. Notre étude rejoint par ailleurs les conclusions de Lucas qui, tout en partant de l'idée étrangère à notre démarche de « système de gouvernement » et de " proconsuls " à propos des représentants en mission, concluait néanmoins à la diversité et à l'adaptation des directives nationales aux conditions locales, Colin LUCAS, La Structure de la Terreur. L'exemple de Javogues et du département de la Loire, Saint-Étienne, CIEREC, Université Jean Monnet, 1990 (édition anglaise, Oxford, University Press, 1971).

(2) Pour la présentation de la sociologie de la petite ville, voir Anne JOLLET, Terre et société en Révolution. Approche du lien social dans la région d'Amboise, Paris, C.T.H.S., 2000. 
sensible lors des mouvements taxateurs qui ont atteint la région à l'automne 1792. Les objets de confrontation révélés à travers les délibérations municipales comme à travers les délibérations du conseil du district se sont multipliés depuis le printemps 1793 (3). Confronté aux réticences de la municipalité face à ses initiatives, le directoire du district envoie en octobre 1793, deux de ses membres à Saumur pour y rencontrer les représentants auprès de l'Armée de l'Ouest. En effet, la Commission centrale des représentants en mission dans l'Ouest siège dans la ville depuis mai 1793. Les deux membres du directoire de district sont parmi les plus actifs : il s'agit du président, Gerboin l'aîné, et d'un homme que les sources désignent comme son ami, un certain Bourlet, choix qui témoigne de l'importance politique accordée à la démarche. Celle-ci est suivie de l'établissement d'un comité révolutionnaire à Amboise par «les représentants du peuple près l'armée de l'Ouest réunis à Saumur " le 5 brumaire an II (26 octobre 1793) (4). Ce comité, au cœur des discordes locales, sera le plus souvent dénommé le comité Richard. L'arrêté contient la liste des huit personnes devant composer ce comité. Il s'agit de personnes, bien connues à Amboise pour leur engagement en faveur de la Révolution du 20 juin (5), mais qui étaient probablement inconnues des représentants et dont les noms ont été proposés par les deux commissaires qui se trouvent eux-mêmes parmi les huit membres.

L'épisode constitue une coupure dans la vie politique locale. En effet, à la suite de cette intervention sollicitée, les deux sociétés populaires sont fondues en une seule, la «Société des sans-culottes d'Amboise », les anciens notables sont exclus de la nouvelle société, et l'ancien maire est emprisonné (6). Ces décisions suscitent immédiatement de vives protestations qui aboutissent à la venue à Amboise, dès le 4 novembre, des représentants Richard et Merlin.

Le déroulement de ces événements met en évidence la complexité du jeu concret des pouvoirs, du local et du national. Il montre comment les divergences politiques locales peuvent prendre appui sur les représentants en mission, habilement sollicités par les uns et les autres en fonction d'accointances politiques, d'une réputation des représentants qui constitue une forme de culture politique des élites locales. Les deux commissaires se sont

(3) Voir Anne JOLLET, «Approches d'un microcosme politique de l'an II à l'an III : l'exemple d'Amboise en Touraine ", 120 c Congrès national des sociétés historiques et scientifiques, Aix-en-Provence, 1995 ; dans M. VoveluE dir., Le toumant de l'an III. Réaction et Terreur blanche dans la France révolutionnaire, Éd. du C.T.H.S., 1997, pp. 325-337.

(4) AD Indre-et-Loire, 3 L 28, Délibérations et arrêtés du département, des représentants du peuple concernant le district d'Amboise, 5 février an II-24 brumaire an III.

(5) Voir Anne JoLLET, « La temporalité d'un ardent révolutionnaire à travers une publication autojustificative de l'an II ", dans Pour la Révolution française, Mélanges publiés en hommage à Claude Mazauric, Rouen, 1998, pp. 57-62.

(6) AD Indre-et-Loire, 3 L 6, Délibérations du conseil général du district d'Amboise. 
adressés à Richard et à Choudieu tandis que les représentants rassemblés à Saumur à un moment ou un autre au cours de l'automne ont été nombreux, parfois dix dans le cadre du conseil de guerre établi à Saumur (7). Le Comité de salut public avait en effet décidé la réunion à Saumur en septembre d'un conseil de guerre composé de représentants et de généraux pour trancher sur le plan à adopter pour la guerre en Vendée. Dans cette affaire, Choudieu a un rôle majeur et on peut faire l'hypothèse que son opposition avec Philippeaux depuis le printemps fait partie des connaissances des acteurs amboisiens, que les deux représentants sont connus pour leur position lors du procès du roi, comme montagnards et comme hostiles au «modérantisme ». L'arrêté est pris le 26 octobre alors que Choudieu quitte Angers le 28 octobre et rentre alors à la Convention. Par ailleurs, au même moment, un autre représentant en mission est présent plus près d'Amboise : il s'agit de Guimberteau, envoyé à Tours début octobre pour la levée des chevaux dans cinq départements autour de l'Indre-et-Loire. Guimberteau a même reçu la charge de procéder à l'épuration des autorités départementales du Loir-et-Cher et d'Indre-et-Loire (8). Il a épuré les autorités municipales à Blois et à Tours d'une façon qui lui attire d'ailleurs les reproches de la Convention. Il devait partir vers l'armée des Côtes de Cherbourg dès novembre, mais finalement il reste jusqu'en mars 1794 à Tours.

Ce représentant ignoré, volontairement le plus probablement, des Jacobins locaux va pourtant, malgré eux, jouer un rôle important dans la vie politique locale. Les délibérations de la municipalité en particulier relatent les échanges de renseignement, les demandes et les adresses à Guimberteau dès son arrivée.

\section{Sollicitation par les acteurs locaux des instances nationales}

Cependant les autorités locales ne limitent pas leurs interventions vis-àvis des pouvoirs nationaux à la seule interpellation des représentants présents. Ils envoient fréquemment des courriers mais aussi, dans les moments de fortes tensions, des commissaires à Paris. Ce fut le cas notamment à l'automne 1792 lorsque les autorités de district ont été accusées par

(7) Les représentants Richard et Choudieu jouent le rôle essentiel dans cette affaire. Choudieu est un homme du lieu puisque, né à Angers, il y a été procureur du roi au présidial. Il a depuis été élu député du département du Maine-et-Loire à la Législative puis à la Convention. Il a été envoyé dans son département pour surveiller la levée des $\mathbf{3 0 0} 000$ hommes. Lors de l'explosion des troubles en Vendée, il fait partie de ceux qui, installés à Saumur, sont chargés d'organiser les forces républicaines. Il est possible que Gerboin aîné, juriste lui-même, ait connu Choudieu, avant sa mission. Richard est lui élu de la Sarthe, département voisin, également dans les deux assemblées. Il a été envoyé en mission avec Choudieu pour l'organisation de la levée.

(8) Les délibérations du conseil du département mentionnent son arrivée le 17 octobre. 
le ministre de l'Intérieur Roland et par le département d'avoir soutenu les émeutiers au moment de l'arrivée des taxateurs de la Beauce à Amboise. C'est à nouveau le cas en décembre 1793. Le 11 frimaire an II (1er décembre 1793), les autorités constituées et les commissaires de la société populaire sont " réunis pour délibérer sur les moyens de venir au secours du district totalement dénué de subsistances " (9). Cela fait des semaines que les autorités multiplient les actions pour lutter contre la famine qui menace, contre une situation de très grande misère dans cette région toujours déficitaire en blés : les autorités constituées se plaignent du fait que leurs délibérations sont constamment interrompues par des citoyens en pleurs qui viennent dire leur misère. Elles décident d'envoyer deux commissaires auprès de la Convention nationale pour lui faire connaître «l'état des besoins pressants du district d'Amboise". Ces commissaires sont chargés de faire les démarches nécessaires «soit auprès de la commission des subsistancés, soit auprès du ministre de l'Intérieur ». Mais ils sont aussi invités à se présenter " tant à la Commune de Paris qu'à la Société des Jacobins pour les assurer des sentiments fraternels que ce district leur a voué ». La Société des sansculottes républicains est invitée à nommer à son tour deux commissaires. La question des subsistances est certes obsédante dans cette région qui doit faire venir ses grains du Nord, grains dont l'acheminement est rendu difficile par la rupture des ponts sur la Loire. Mais la mission a aussi une portée politique : il s'agit de faire comprendre la situation politique créée par la dernière épuration et de se faire des appuis alors que la contestation est forte localement. L'importance politique du déplacement est confirmée par le fait que parmi ces commissaires se trouve un individu dont l'implication dans la vie politique locale est très importante depuis le début de la Révolution et encore plus depuis le 10 août. Cet individu, Gerboin aîné, membre du directoire du district, est l'un des deux hommes qui sont allés à Saumur, et il est le président du Comité révolutionnaire issu de cette démarche, donc un personnage de premier plan de la radicalisation démocratique de l'an II (10).

L'intervention est d'autant plus nécessaire que les partisans de l'ancien maire s'expriment à travers le conseil général de la commune, demandent des comptes, s'adressent à leur tour au représentant en mission. Le 1er nivôse an II ( 21 décembre), le conseil général de la commune rappelle la demande qui a été faite de l'envoi des motifs de l'arrestation du maire au représentant du peuple Guimberteau. Mais le « comité révolutionnaire de la an II.

(9) $\mathrm{AD}$ Indre-et-Loire, 3 L 6, Délibérations du conseil général du district d'Amboise, 11 frimaire

(10) Anne JOLLET, «Approches d'un microcosme politique de l'an II à l'an III : l'exemple d'Amboise en Touraine ", op. cit. 
section d'Orient, malgré des courriers adressés à lui à ce sujet ", ne répond pas. Le Conseil fait une dernière demande, menaçant en cas de refus de s'adresser au Comité de sûreté générale et à Guimberteau.

\section{Les représentants à l'initiative de l'intervention dans les rapports de force locaux}

La situation d'Amboise est effectivement connue de Guimberteau. Le lendemain, 2 nivôse, le général Descloseaux, commandant la réserve à Tours, et Rouhière, secrétaire du représentant en mission, ici envoyé par ce dernier, sont présents à la maison commune d'Amboise. Il n'est pas question de la précédente arrestation, mais l'esprit public des Amboisiens est l'objet de la curiosité des visiteurs : "Le général a dit qu'il venait voir ses frères d'Amboise et s'assurer de leur disposition à servir la République et qu'il avait donné ordre de rassembler les deux bataillons pour se rendre au temple de la raison et y faire l'inspection des hommes et des armes » (11). Le citoyen Rouhière a remercié la commune au nom de Guimberteau «pour l'empressement avec lequel les citoyens avaient leur souliers". Le général et le commissaire du représentant sont ensuite sortis, suivis des membres du conseil et tous se sont rendus à la place d'armes. Là, le général a harangué les troupes puis on s'est déplacé au temple de la raison où a eu lieu une scène de déchristianisation : "le peuple apercevant des restes d'images et statues rassemblées par le ci-devant fanatisme et la superstition malgré toute la surveillance que faisait la municipalité pour détruire le fanatisme [...] les a brisés spontanément en a purgé entièrement le temple déjà consacré à la raison [...] un feu vraiment civique a épuré le temple, les citoyens des deux sexes ont dansé tout autour au son des instruments qui jouaient les airs immortels de l'hymne de la liberté et de la Carmagnole »(12). La même scène s'est produite dans les deux autres temples.

Après cette manifestation de son patriotisme et de son dévouement au représentant en mission, le même jour, le conseil de la commune décide de l'envoi de deux commissaires (les mêmes personnes dans les deux missions) à Tours et à Paris auprès de la Convention pour réclamer la libération du maire.

La tension politique locale reste grande : la société des sans-culottes intervient à plusieurs reprises auprès de la municipalité pour l'inciter à agir, lui faire des remarques sur son attitude.

(11) Archives communales d'Amboise, D 10, Délibérations du conseil général de la commune, 2 nivôse an II.

(12) Ibid. 
Coup de théâtre : le 5 nivôse, les frères Gerboin, le président du comité de surveillance révolutionnaire et son jeune frère, également membre de l'administration de district, ainsi que d'autres membres du comité, dit comité Richard, sont arrêtés et incarcérés à la citadelle d'Amboise. L'ancien maire est, lui, libéré et réintégré dans ses fonctions le 14 nivôse ( 3 janvier 1794), ceci par un arrêté de Guimberteau du 13 nivôse.

Ledit arrêté est transcrit sur les registres de délibérations du conseil général de la commune et les membres ont « unanimement témoigné leur satisfaction de la justice rendu au citoyen maire " (13). Les élus municipaux rappellent dans les jours qui suivent ce que cette victoire doit selon eux à leur démarche à Paris et à Tours. Lorsque les deux commissaires viennent présenter le mémoire de leurs frais de voyage à Paris, le conseil estime « que ce succès a contribué à déjouer une faction qui opprimait les citoyens et à rendu à la commune la paix et la tranquillité et a rétabli la fraternité entre tous les citoyens " (14).

Dans ce nouvel épisode, l'arrêté du représentant est le fruit d'une interpellation par les acteurs locaux et, s'il est difficle de faire la part du poids respectif du recours parisien et du recours direct auprès du représentant en mission dans la conclusion de cette affaire, l'intervention du pouvoir central y apparaît bien comme déterminante, mais plus comme un outil au service d'une fraction des pouvoirs locaux que comme pouvoir autonome.

À la suite de ces événements, Guimberteau se rend lui-même à Amboise. Il arrive le matin du 1er pluviôse (le 20 janvier). Il est accompagné du général Descloseaux et de son secrétaire Rouhière. C'est le maire qui les reçoit. Guimberteau demande si la municipalité n'a pas quelques réclamations à lui soumettre. Le maire mentionne "la plus grande disette de subsistance ». Le représentant s'adresse ensuite au peuple pour lui demander s'il a des plaintes contre ses magistrats. «Aucune voix ne s'étant élevée le représentant a félicité le peuple d'avoir des magistrats vertueux, et les magistrats d'avoir la confiance du peuple» (15). Les délibérations de la municipalité précisent que le représentant «[...] n'a pas voulu quitter la maison commune sans donner avec la plus grande effusion de cœur l'accolade fraternelle au maire en le chargeant de la rendre à tout ses concitoyens ».

Il s'agit évidemment d'un geste politique fort, tendant à consolider le rôle du maire. La confirmation du sens politique de cette intervention est apportée par l'hostilité qu'affiche une partie des sans-culottes d'Amboise vis-à-vis de Rouhière et, de façon plus prudente, vis-à-vis de Guimberteau lui-même.

(13) Ibid.

(14) Archives communales d'Amboise, D 10 Délibérations du conseil général de la commune, 25 nivôse an II (14 janvier 1794).

(15) Ibid., 1er pluviôse an II (20 janvier 1794). 
Recours des acteurs locaux aux instances nationales contre les interventions des représentants

Les partisans du comité Richard ne sont en effet pas restés inertes non plus et se sont adressés directement à la Convention pour dénoncer les manœuvres des modérés à Amboise. Or la position de Rouhière n'est pas inattaquable puisque pèse sur lui le soupçon d'avoir été un partisan de Dumouriez. L'écho d'une campagne de dénigrement à l'encontre de Rouhière apparaît également dans la suite des délibérations municipales, ce qui témoigne là aussi de la circulation de l'information ainsi que du fait que les menées des anciens membres du comité étaient bien connues de la municipalité. Un membre du conseil déclare que «le conseil n'ignore pas que le citoyen Rouhière avait été calomnié en cette commune par ces hommes dont l'opinion publique venait enfin de faire justice, que cependant tous les citoyens savaient comment Rouhière lors de son voyage en cette commune avec le général avait porté des coups assurés aux restes du fanatismes et à l'intrigue qui dominait encore à cette époque... » (16). Il est décidé que l'expression de l'estime des citoyens sera consignée dans les registres et qu'une expédition en sera faite à Rouhière, puisque «les intrigants de cette commune qui avaient calomnié le représentant du peuple Guimberteau lui-même avait également calomnié son secrétaire parce que la calomnie est leur élément ». Guimberteau lui-même a en effet été obligé de justifier son action devant le Comité de salut public qui a conclu au fait que les citoyens libérés par Guimberteau avaient été arrêtés par "esprit de parti ».

Dans les jours qui suivent ( 3 pluviôse an II -22 janvier), le conseil municipal réorganise les comités de surveillance révolutionnaires qui sont constitués dans les deux sections de la commune. Les arguments mis en avant sont le trop faible nombre de la population, le degré de parenté prohibé et le fait que le choix de 24 membres se fait aux dépens des autres administrations, d'autant plus que beaucoup «quoique bons républicains et braves sans-culottes ne savent ni lire ni écrire ».

\section{Appel au soutien du représentant en mission contre les interpellations des instances nationales}

Il n'est rien dit dans les délibérations municipales des places vacantes du fait de l'incarcération d'un certain nombre de membres du comité de la section d'Orient (qui correspond aux faubourgs de la ville), ni de l'hostilité qui perdure entre ce comité et la municipalité. La municipalité rapporte 
donc son arrêté du 6 brumaire (27 octobre 1793) qui prenait acte de la création du comité révolutionnaire par Richard et Choudieu et soumet ses observations à Guimberteau qui «sera invité de réduire les deux comités de surveillance révolutionnaire en un seul pour cette commune ».

La démarche témoigne de la prudence de la municipalité qui cherche à bien s'assurer l'appui du représentant et à s'abriter derrière son autorité. Dans le même sens, le conseil décide dans les jours qui suivent (5 pluviôse 24 janvier) de s'adresser à nouveau à la Convention pour l'instruire « de l'épuration faite par le représentant du peuple Guimberteau du conseil général de la commune le 1er de ce mois et de lui faire une adresse qui lui peindrait les sentiments du conseil général sur la Révolution et ceux manifestés par tous les citoyens lors du court séjour des représentants du peuple». Il s'agit bien de se justifier face à d'éventuelles critiques, ce qui témoigne à nouveau du fait que le pouvoir du représentant ne se substitue pas totalement dans l'esprit des élus locaux - ne se superpose pas tout à fait - avec celui de la Convention à Paris. On sait que celle-ci peut être informée par d'autres voix, que d'autres représentants en son sein peuvent être sensibles à d'autres renseignements, à d'autres influences, à d'autres tendances politiques.

Le projet d'adresse suit. Il commence par ces mots « Notre 1er devoir après avoir subi l'épuration révolutionnaire est de vous adresser l'expression [...] », et il y est question de la « presque universalité de nos concitoyens ».

La responsabilité du représentant en mission est mise en avant en termes emphatiques qui dramatisent la scène de la visite par rapport au compte rendu qui en est donné dans les délibérations municipales et dans celles de l'administration de district.

«Représentants, nous n'oublierons jamais [...] la journée du 1 er pluviôse où un de vos collègues revêtu de pouvoirs illimités, le citoyen Guimberteau, est venu exercer au milieu de nous la puissance nationale, le crime aurait tremblé à son aspect imposant, la vertu n'avait rien à craindre, nous avons entendu avec sécurité le représentant du peuple interroger ce bon peuple sous la conduite de ses magistrats depuis la Révolution et aucun de nous ne s'est trouvé avoir démérité.

Alors mille cris répétés de vive la République, vive la Convention nationale, vive la montagne, vive Guimberteau, vive les sans-culottes, ont bien vengé le maire d'Amboise mis en arrestation par l'intrigue et le conseil général de la commune de quelques calomnies lancées par la malveillance. "

Le tableau dressé est celui, conventionnel, du plus grand enthousiasme, de la plus grande unanimité : "enthousiasme patriotique "... au point que le représentant «a pu compter autant de sans-culottes que de têtes». Enthousiasme autour de Guimberteau : "on se pressait autour de 
Guimberteau et l'on n'ententait plus qu'un seul cri vive la République, la République ou la mort ».

Il s'agit bien de renforcer la légitimité de l'intervention de Guimberteau, contestée localement, contestée aussi à Tours où l'épuration lui a attiré les reproches de Barère qui l'a contraint à réintégrer certains des destitués.

Contre les décisions d'un représentant, la sollicitation d'autres représentants en mission

Cette prudence s'explique d'autant mieux qu'à Amboise comme à Tours, l'opposition à l'épuration menée par Guimberteau ne désarme pas, ce qui témoigne à nouveau de la force des antagonismes locaux et des limites de l'autorité du représentant face à la détermination des acteurs locaux.

Le registre du comité de surveillance épuré permet de savoir qu'une pétition a circulé à Amboise, réclamant à Ichon, représentant du peuple à Tours, la mise en liberté des frères Gerboin et des membres du comité révolutionnaire (17). Le texte de cette pétition à propos de laquelle on interroge un certain nombre d'Amboisiens n'est pas recopié dans le registre, mais il y est précisé qu'elle contient des "expressions dures et calomnieuses » contre des citoyens qui sont traités de "calomniateurs, aristocrates et modérés ». Cette pétition devait être expédiée au représentant Ichon, lui aussi envoyé auprès de l'armée de l'Ouest et lui aussi chargé d'épurer les différentes autorités en Indre-et-Loire. Cette activité s'organise notamment autour du directeur de la poste aux lettres chez qui se font des rassemblements régulièrement dénoncés par les membres du conseil de la commune, autre témoignage de l'importance de la circulation de l'information dans ces rapports de force locaux et notamment de la circulation entre la province et Paris.

En germinal an II (mars 1794), dans l'affaire qui oppose l'ancien directeur de la poste aux lettres au nouveau comité de surveillance révolutionnaire et au maire, on constate l'obstination des résistances des acteurs locaux. Le directeur a été démis de ses fonctions. Le courrier a été saisi. Une lettre est ouverte. Pottet, l'ex-directeur, l'examine puis refuse de remettre la lettre. Pottet «a déclaré ne vouloir la remettre et être dans l'intention de l'envoyer au représentant du peuple Francastel et a exigé notre signature au dos de ladite lettre ce qu'avons fait et le citoyen Pottet qui avait déclaré vouloir remettre la lettre l'a retenue par devers lui et a déclaré ne vouloir signer de ce interpellé » (18). Le citoyen Pottet est reconduit en prison à la

(17) $\mathrm{AD}$ Indre-et-Loire, $1 \mathrm{~L} 24$, Délibérations du comité de surveillance.

(18) Archives communales d'Amboise, G IV 123, comité de surveillance. 
citadelle mais il ne se dessaisit pas de la lettre. Dans les jours qui suivent, l'agent national de la commune d'Amboise se rend avec un officier municipal chez l'épouse du directeur qui refuse de les laisser rentrer et accuse l'officier municipal d'avoir volé une lettre. L'officier dénonce immédiatement cette «calomnie » à Francastel.

Le 14 floréal an II, un «arrêté des représentants du peuple Francastel, Ichon, Hentz, portant entre autres dispositions que le directeur de la poste aux lettres de cette commune sera mis en liberté et reprendra de suite ses fonctions de directeur de la poste aux lettres, faire remettre par le citoyen Sanche le jeune, qui avait été nommé provisoirement pour remplir ces fonctions, tous les registres, biens et papiers énoncés au procès-verbal. Sanche accepte immédiatement » (19). La démarche a été menée par le nouveau maire Deslandes qui signe, ainsi que Pottet lui-même.

On a ici affaire à un nouvel épisode dans lequel on constate une des multiples façons dont les oppositions locales utilisent la présence de différents représentants dans la région pour utiliser à leur profit leur pouvoir d'intervention dans la vie locale.

\section{Les attaques des acteurs locaux amènent les représentants à rendre des comptes}

La poursuite de la lutte se fait aussi par l'interpellation par les acteurs locaux de l'un ou de l'autre des pouvoirs parisiens. Gerboin l'aîné, l'ancien président du comité révolutionnaire, a, dès le 10 pluviôse (29 janvier), écrit aux Jacobins de Paris pour donner sa version des faits. Pour lui, il s'agit d'un épisode de la lutte entre «aristocrates » et « républicains » et d'une défaite de ces derniers. Il ajoute que «la religion du représentant du peuple Guimberteau a été surprise par l'aristocratie » et que la société populaire n'est peuplée que de «suspects» dont «nous avons envoyé la liste au Comité de sûreté générale » (20).

Cette lettre n'est pas restée sans effet puisque la Convention nationale elle-même discute de la situation d'Amboise dans sa séance du 5 ventôse (24 février) et Guimberteau doit répondre à une demande d'explication du Comité de salut public. À son tour, il répond par lettre du 8 ventôse an II (26 février 1794) que la Convention nationale a été égarée au sujet des frères Gerboin : «Dès les premiers moments de mon arrivée à Tours [écrit-il] la commune d'Amboise était déchirée par une faction oppressive qui 
gouvernait par la terreur, comprimait le patriotisme et lançait des mandats d'arrêt contre les meilleurs patriotes. Cette faction avait pour chefs les frères Gerboin dont l'aîné était membre d'un comité révolutionnaire " (21). Il reprend l'idée des opposants locaux aux Gerboin, de l'existence d'un triumvirat tyrannisant l'opinion et justifie lui-même son action par les demandes locales. Il poursuit : "Chaque jour, je recevais des pétitions, des réclamations de nombreuses victimes de la faction; elles se multipliaient au point que, craignant un soulèvement général, je crus de mon devoir de chercher à m'éclairer [...] ». Il explique que les Gerboin ont été exclus par épuration et non par lui et qu'il n'a « ordonné aucune incarcération ». C'est le comité de surveillance de la commune qui «crut nécessaire de s'assurer de la personne des Gerboin » et «ils furent par son ordre seul et sur les dénonciations du peuple, sans m'en prévenir, traduits à la citadelle d'Amboise » (22).

Cette façon de décrire son action montre que le représentant cherche surtout à se présenter comme le défenseur de la paix civile, comme celui qui cherche à apaiser les tensions - ce qui fut effectivement le cas dans nombre de cités (23)-, un outil pour la population plus que l'initiateur d'une politique propre. Ceci témoigne aussi de l'efficacité des moyens dont peuvent disposer les adversaires de la politique menée par un représentant pour faire connaître leur opposition, en cherchant notamment à informer euxmême l'Assemblée, concurremment au représentant, sur ce qui se passe localement. La réponse prudente de Guimberteau montre que les critiques ne restent pas sans écho et que dans sa lutte contre les exagérés, il risque de se voir lui-même taxer de modérantisme.

Lorqu'elle félicite la Convention nationale à la suite de l'élimination des hébertistes, la municipalité d'Amboise fait le parallèle entre la situation locale et la situation nationale, montrant que les affrontements politiques parisiens sont bien présents dans les consciences amboisiennes et qu'il s'agit de s'inscrire dans ce rapport de force. En même temps, le texte redonne au représentant sa responsabilité, de même qu'il montre à travers le rappel d'un dernier événement survenu à Amboise, le prestige et l'autorité des représentants. Le texte rappelle en effet que "les alarmes sur les subsistances étaient partout le mot d'ordre des scélérats; des intrigants de notre commune avaient publié que la municipalité épurée par Guimberteau était destituée par un autre représentant du peuple près de l'armée de l'Ouest [...] » (24). L'allusion renvoie à la présence à Amboise, au mois de mars, d'un individu qui dit tenir ses pouvoirs du représentant Francastel.

(21) Alphonse AUlard, Recueil des Actes du Comité de salut public, Paris, 1889-1933.

(22) $l d$.

(23) Voir, par exemple, l'action du représentant Lefiot à Clamecy (Nièvre), dans Nicole BossuT, "Terreur à Clamecy. Quelques réflexions ", A.H.R.F, n 311, janvier-mars 1998, pp. 49-77.

(24) Archives communales d'Amboise, D 11, délibérations du conseil général de la commune. 
L'individu est notamment en contact avec le directeur de la poste aux lettres et, selon les dires de la municipalité, il aurait tenté de provoquer une nouvelle épuration des autorités constituées en s'appuyant sur deux anciens membres, non incarcérés, du comité révolutionnaire.

L'insistance de la municipalité à dénoncer ces démarches traduit une réelle pression de ses opposants qui peuvent effectivement s'appuyer maintenant sur de nouveaux représentants et sur la crainte du Comité de salut public de voir triompher les «modérés». De fait, le 14 floréal an II (1er mai), un arrêté des représentants du peuple Francastel, Ichon, Hentz, en mission auprès des armées de l'Ouest, amène à une nouvelle épuration des autorités constituées. La société populaire est dissoute et une nouvelle société est constituée. Un nouveau conseil révolutionnaire de la commune d'Amboise est formé et plusieurs membres de l'ancien comité révolutionnaire reviennent dans l'administration de district.

Couronnement de ce retournement du rapport des forces, un décret de la Convention du 4 prairial (24 mai) rétablit les frères Gerboin dans leurs fonctions. L'ancien maire est à nouveau arrêté. La nouvelle opposition, soutien de l'ancien maire, reprend la lutte et les nouvelles autorités de district désignent des «malveillants qui ne cherchent qu'à fomenter des troubles en condamnant avec une affectation marquée les épurations faites en cette commune par Hentz et Francastel ». De même, le représentant Ichon se donne la peine de passer à Amboise le 22 mai, d'y prendre la parole pour dénoncer l'existence d'une "pétition tendant à enlever aux autorités épurées la confiance du peuple ». Il tonne contre la «mollesse du Comité de surveillance ». Des scellés sont mis sur les biens de l'ancien maire. Ces autorités épurées resteront néanmoins au pouvoir jusqu'en fructidor an II où une nouvelle épuration menée par un nouveau représentant, Brival, écartera définitivement les sans-culottes et les Montagnards les plus prononcés, les Gerboin et leurs amis, de la vie politique locale (25). L'ancien maire le redevient encore une fois le 11 prairial an III (30 mai 1795).

\section{Conclusion}

À travers cette présentation de l'action des représentants en mission vue au niveau de la vie politique locale, nous sommes amenés à constater combien l'intervention de ces représentants est un élément parmi d'autres dans le jeu des forces qui animent cette vie politique. Leurs interventions n'arrivent pas dans un milieu inerte, mais dans un milieu bien informé des

(25) Les frères Gerboin se désignent eux-mêmes par le terme de « Montagnards ». Dans le mémoire qu'il fait imprimer pour sa défense le 17 ventôse an II, Gerboin le jeune se qualifie de « Montagnard zélé " (cf. Anne JoLLET, « La temporalité d'un ardent révolutionnaire... », op. cit.). 
grands clivages nationaux et lui-même travaillé, divisé par ces débats. Ces repésentants sont sollicités, mis en concurrence entre eux, mis en concurrence avec d'autres pouvoirs, notamment avec le pouvoir dont ils tiennent leur légitimité, la Convention nationale. Leur autorité est réelle, mais les divers groupes de pression locaux cherchent constamment à l'instrumentaliser. Cependant ces représentants ont eux aussi leur personnalité, leur point de vue, sont l'expression d'un moment du gouvernement révolutionnaire dont ils cherchent à imposer la marque. La persistance des affrontements autour de choix politiques de plus en plus nets à Amboise témoigne du fait que la Terreur ne terrorise pas les acteurs politiques locaux qui savent bien jouer des divisions au sein du gouvernement révolutionnaire et de la diversité de sensibilité politique des représentants.

Loin d'apparaître comme des «proconsuls » omnipotents, les représentants sont ici pris dans une circulation de l'information dont ils sont loin de maîtriser tous les éléments, dans une circulation qui en grande partie leur échappe, dans une circulation des hommes qui, de même, n'est pas seulement leur fait, dans une diversité des pouvoirs qui jouent, parfois à leurs dépens, comme des contre-pouvoirs. Ils sont eux-mêmes l'objet d'une surveillance, surveillance attentive de la Convention, surveillance des Jacobins, surveillance du centre sur ses émissaires, mais surveillance relayée par les pouvoirs locaux qui ne se privent pas de jouer de la multiplicité des pouvoirs pour asseoir le leur.

À l'issue de cette étude de la fonction et des usages des représentants en mission dans la vie d'une petite ville, il semble donc prudent de se méfier des réifications opposant un centre et des périphéries, en particulier un "pouvoir parisien », abstraction globalisante, désignant un pouvoir unifié opposé à la diversité et à la matérialité du local, du provincial. On voit bien à travers la vie amboisienne pendant les quelques mois de l'an II comment se rencontrent ici des manifestations des pouvoirs divers du centre parisien et des pouvoirs provinciaux, et combien c'est dans le heurt, les rivalités, les ajustements permanents entre ces pouvoirs que se prennent les décisions et que se dessinent non sans à-coups les formes de la politique locale.

Anne JOLLET

Gerhico

Université de Poitiers

145, rue de Tolbiac

75013 Paris 